\section{Index of Species}

Page numbers in italics refer to the subjects of the papers

Adalia bipunctata, 292

Aedes albopictus, 25, 26, 173-181

hendersoni, 180

triseriatus, 180

Alces alces, 225

Allium schoenoprasum, 253-256, 257-261

Amphicarpaea bracteata, 27-31

Amsinckia grandiflora, 37

Anolis grahami, 173

Antirrhinum majus, 237, 256

Brassica, 452

Caledia captiva, 39-46

Capreolus capreolus, 221-227

Cervus elephus, 144, 225

Cheranthus cheiri, 37

Clarkia, 247, 263

amoena, 237, 245, 246

subsp lindleyi, 245

arcuata, 237, 245

bottae, 245

davyi, 245

gracilis, 237-246

subsp. albicaulis, 237, 245, 246

gracilis, 237-246

sonomensis, 237-246

tracyi, 237, 245, 246

imbricata, 245

lassenensis, 237, 246

nitens, 245

purpurea, 245

rubicunda, 237

xantiana, 237

Costus allenii, 260

laevis, 260

Culiseta longiareolata, 21-26

Dactylis glomerata, 375

Dahlia variabilis, 375

Dama dama, 225

Dasyuroides byrnei, 77-85

Dasyurus hallucatus, 77-85 viverrinus, 77-85

Dipcadi serotinum, 247-252

Drosophila, 21, 25, 47, 58, 59, 116, 135, 157, 229, 234, 263, 305, $343,421,423$

Drosophila ananassae, 269-272

arizonensis, 299-304

hydei, 364

mauritiana 44, 299

melanogaster, 7-14, 25, 55-60, 101-107, 119-123, 129-137, 157, 158(bk. rev.), 205-212, 213-219, 273-281, 292, 299,

$304,335-346,355-366,367-374,403-414,415,427-433$

mojavensis, 299-304

pavani, 269, 271, 272

persimilis, 269, 271, 272

obscura, 59

pseudo-obscura, 59, 269, 271, 272, 301

sechellia, 299

simulans, 44, 129, 299, 403-414

subobscura, 269, 272, 284

virilis, 25

willistoni, 364
Eliomys quercinus, 125-127

Endymion non-scriptus, 256

Eudyptula minor, 69-76

albosignata, 69

iredalei, 69

variabilis, 69

Euplectes progne, 291

Euthystira brachyptera, 383-386

Fagus sylvatica, 91-100

Felis domesticus, 144

Gammarus oceanicus, 229

Gilia achilleifolia, 257

Glycine argyrea, 443

Godetia, 245

Gossypium, 237

guppyfish see Poecelia

Haplopappus spinulosus, 375

Helianthus, 247

Homo sapiens, 144

Hordeum, 229, 247

Hordeum vulgare, 65, 148

Hyacinthoides non-scripta, 256

Hyla cinerea, 44

gratiosa, 44

Ipomoea purpurea, 237, 256, 261, 439, 443

Isoodon obesulus, 77-85

macrourus, 77-85

Isotoma, 47

Lasiorhinus latifrons, 77-85

Lepidodactylus lugubris, 463-467

Lilium callosum, 267

Little Blue Penguin see Euclyptula

Locusta, 41

migratoria, 203, 401

Lythrum salicaria, 37

marsupials, 77-85

Mazama americana, 144, 225

Medicago falcata, 375

sativa, 375

Megadyptes antipodes, 69-76

Microtus agrestis, 144

Mimulus guttatus, 443

Mus, 45

Musca autumnalis, 173

Muscari comosum, 251

Myotis californicus, 144

velifer, 144

Myrmeleotettix maculatus, 267, 383-386

Nemesia strumosa, 237

Nicotiana rustica, 1-5, 147 tabacum, 1-5

Odocoileus virginianus, 139-146, 229

Oenothera, 47

Oryctolagus cuniculus, 173 
Panicum maximum, 162

Papaver rhoeas, 237

Perognathus californicus, 144

Peromyscus maniculatus, 144, 229

Perameles gunnii, 77-85

nasuta, 77-85

Petunia, 237

Pharbitis nil, 237

Phascolarctos cinereus, 77-85

Phleum pratense, 375

Phlox drummondii, 260

Pinus sylvestris, 260 taeda, 260

Poecelia reticulata, 284 sphenops, 463, 467

Primula sinensis, 237

Proechimys guarirae, 144

Pseudotsuga menziesii, 260

Pycnogaster cucullata, 197-204

finotti, 179-204

graellsi, 197-204

inermis, 197-204

sanchez-gomezi, 197-204

Rana esculenta, 463

Rangifer tarandus, 144, 225

Rumex acetosa, 109-117, 263-268

arifolius, 115

rye, $47-54,455$

Sarcophilus harrisii, 77-85

Schistocerca gregaria, 387-401

Scilla autumnalis, 109, 116, 251

Secale cereale, 47-54

Senecio squalidus, 36,37

vulgaris, 15-20, 33-38, 257, 295-298

Sminthopsis crassicaudata, 77-85

murina, 77-85

Solanum chacoense, 183-191

phureja, 184

rybinii, 191

tuberosum, 147-153, 183-191, 375

Sorghum, 247

bicolor, 380

Speonomus colluvii, 321-327

delarouzeei, 325

hydrophilus, 321-327

zophosinus 321-327

Sphaeroma rugicauda, 229-235
Stephanomeria, 247

Stercorarius parasiticus, 283

Stereopleuras martorelli, 197

Stethophyma grossum, 386

Synchytrium decipiens, 27-31

Talpa occidentalis, 125-127

Taraxacum, 161-171

aristum, 161-171

bessarabicum, 161-171

brachyglossum, 161-171

pseudo-hamatum, 161-171

pyropappum, 161-171

sect. vulgana, 161-171

serotinum, 161-171

ungulobum, 161-171

Thomomys bottae, 144

Tolmiea menziesii, 375-382

Tragopogon mirus, 375

miscellus, 375

$6 \times$ triticale, $455-461$

Triticum alloplasmic lines.

Chinese Spring, 87-88

Selkirk, 87-88

Triticum (genus) $87-90$

aestivum, 87-90, 455

aucheri, 87-90

bicorne, 87-90

longissima, 87-90

monococcum, 88

searsii, $87-90$

sharonense, 87-90

speltoides, $87-90$

tauschii, 87-90

timopheevi, 87-90

turgidum, 87-90, 455-461

uratu, 87-90

Tulipa australis, 109, 116

Turnera ulmifolia, 375

Vandiemenella, 266

Vombatis ursinus, 77-85

Wapiti, 225

White-tailed deer see Odocoileus virginatus

Zea mays, 115, 267, 380

Zoarces viviparus, 229 